\title{
Die sittliche Einbettung der Wirtschaft. Von der Effizienz- und Differenzierungstheorie zu einer Theorie wirtschaftlicher Felder
}

\author{
MPIfG Journal Article \\ Jens Beckert Jens Beckert: Die sittliche Einbettung der Wirtschaft: Von der Effizienz und Differenzierungstheorie zu einer Theorie \\ wirtschaftlicher Felder. In: Berliner Journal für Soziologie 22(2), 247-266 (2012). Springer VS \\ The original publication is available at the publisher's web site: http://dx.doi.org/10.1007/s11609-012-0187-y \\ The MPIfG Journal Articles series features articles by MPIfG researchers and visiting scholars published in peer-reviewed journals. \\ Max Planck Institute for the Study of Societies (MPIfG) Cologne | www.mpifg.de
}

Zusammenfassung: Auch die Wirtschaft moderner Gesellschaften folgt nicht einfach einer ökonomischen Eigengesetzlichkeit, sondern konstituiert sich aus ihrer Einbettung in ein Gefüge sittlicher Werte, Institutionen, Interessen und sozialer Macht. Von welcher theoretischen Grundlage aus lässt sich die Sittlichkeit der Wirtschaft beschreiben? Der Artikel zeigt in kritischer Auseinandersetzung mit der auf der Neoklassik aufbauenden orthodoxen Wirtschaftstheorie, dass es gerade deren normativer Charakter ist, der verhindert, Normen einen angemessenen Platz einzuräumen. Es ist jedoch verfehlt zu denken, dass die Soziologie per se hierzu eine angemessene Alternative anbieten würde. Weder die funktionalistische Differenzierungstheorie noch Teile der Wirtschaftssoziologie werden der Herausforderung gerecht. Vielversprechender erscheint, wirtschaftliche Strukturen mit dem Instrumentarium der Theorie sozialer Felder zu analysieren.

Schlüsselwörter: Wirtschaftssoziologie $\cdot$ Einbettung $\cdot$ Feldtheorie $\cdot$ Differenzierungstheorie $\cdot$ Moralökonomie

\section{The moral embeddedness of the economy. From theories of differentiation and economic efficiency to a theory of economic fields}

\begin{abstract}
The economy of modern societies does not simply operate according to its own inherent laws, but is anchored in a moral and institutional order, interests, and in social power. Upon what theoretical basis can the morality of the economy be described? By critically reflecting on mainstream neoclassical economics, the paper argues that it is the normative character of neoclassical theory which stands in the way of improving our understanding of the normative foundations of the economy. It would be wrong, however, to think that sociology necessarily offers a more adequate alternative. Neither functionalist theories of social differentiation nor certain strands of the new economic sociology are up to the challenge. Using the toolkit provided by the theory of social fields seems to be a more promising way to investigate economic structures.
\end{abstract}

Keywords: Economic sociology $\cdot$ Embeddedness $\cdot$ Field theory $\cdot$

Theory of social differentiation · Moral economy

(C) VS Verlag für Sozialwissenschaften 2012

J. Beckert $(\bowtie)$

Max-Planck-Institut für Gesellschaftsforschung,

Paulstr. 3, 50676 Köln, Deutschland

E-Mail: beckert@mpifg.de 


\section{L'encastrement moral de l'économie. De la théorie de l'efficience et de la différenciation à une théorie des champs économiques}

Résumé: L'économie des sociétés modernes n'obéit pas non plus simplement à des lois purement économiques mais se constitue à partir de son encastrement dans un tissu de valeurs morales, d'institutions, d'intérêts et de pouvoir social. Sur quelles bases théoriques peut-on décrire la morale de l'économie? Cet article montre à travers une réflexion critique sur la théorie économique orthodoxe d'inspiration néoclassique que c'est précisément le caractère normatif de celle-ci qui empêche d'accorder aux normes la place qui leur revient. Il est toutefois faux de penser que la sociologie offre automatiquement une alternative satisfaisante. Ni la théorie fonctionnaliste de la différenciation ni toute une partie de la sociologie de l'économie ne sont à la hauteur de la difficulté. Il apparaît plus prometteur d'analyser les structures économiques avec les outils de la théorie des champs sociaux.

Mots-clés: Sociologie de l'économie - Encastrement - Théorie des champs ·

Théorie de la différenciation · Économie morale

\section{Einleitung}

Welche Rolle spielen normative Orientierungen für wirtschaftliches Handeln? Aus der Perspektive der auf der Neoklassik aufbauenden orthodoxen ökonomischen Theorie muss diese Frage müßig erscheinen. Denn Bezugspunkt der Theorie ist der an seinen individuellen Präferenzen orientierte Akteur, der seinen Nutzen zu maximieren sucht und externe Restriktionen unter den Gesichtspunkten von Kosten und Nutzen, nicht jedoch anhand ethischer Maßstäbe prüft. Die sittlichen Orientierungen der Akteure erscheinen in der Theorie allenfalls als beliebige Geschmäcker, als ethisch unzugängliche private Urteile, die als austauschbare Präferenzen in die Indifferenzkurven eingehen. ${ }^{1}$ Oder aber sie erscheinen als Traditionsbestände, die durch die erzieherische Wirkung des Konkurrenzmechanismus hinweggefegt werden. Dabei wird das zugrunde liegende handlungstheoretische Modell des Homo oeconomicus und der Orientierung an der Steigerung individuellen Nutzens nicht als Theorie der kulturellen Prägung des Handelns in modernen Gesellschaften eingeführt, sondern als Naturkonstante. Der Mensch hat eine natürliche Neigung, ,to truck, barter, and exchange one thing for another“ (Smith 1976, S. 17).

Ironischerweise ist diese Theorie, die die Sittlichkeit aus dem ökonomischen Handeln und aus wirtschaftlichen Strukturen ausgrenzt, selbst eine zutiefst normative Theorie. Als empirische Theorie muss sie als falsifiziert gelten, denn es ist wohl unbestreitbar, dass Akteure nicht durchweg nutzenmaximierend handeln (Elster 1986). Dies wissen Anthropologen, Soziologen und Psychologen seit über einhundert Jahren, und auch in der ökonomischen Theorie bricht sich diese - an sich wenig überraschende - Erkenntnis in Form der Befunde der behavioristischen Ökonomie langsam Bahn, indem etwa auf die Rolle von Fairness im Entscheidungshandeln hingewiesen wird. Die Aussage „Akteure handeln nutzenmaximierend“ muss entweder als eine „Als-ob-Theorie“ (Vaihinger 2007) gelesen

1 Unter Sittlichkeit verstehe ich in diesem Beitrag im Anschluss an Axel Honneth (2011, S. 26) Handlungsweisen, die einen allgemeinen Wert verkörpern. 
werden oder aber als eine auf dem Utilitarismus fußende normative Theorie: „Akteure sollen nutzenmaximierend handeln. "Die ökonomische Theorie ist dann eine Technik, mit deren Hilfe die Akteure in die Lage versetzt werden sollen, Entscheidungen so zu treffen, dass sie ihren Nutzen maximieren. Doch wie in einer Trickkiste wird dieser Normativismus zugleich geleugnet, indem die Modellannahmen als ökonomische Gesetze ausgewiesen werden, die wie Naturgesetze operieren (Böhm-Bawerk 1975).

Unzweifelhaft ist diese ökonomische Theorie ein Erfolgsmodell im Wissenschaftsbetrieb. Der durch die Simplifizierung handlungstheoretischer Annahmen - auf der Grundlage des positivistisch gewendeten Utilitarismus - vollzogene radikale Reduktionismus der Wirtschaftswissenschaften ermöglichte mathematische Modellierungen komplexer Zusammenhänge, mit denen sich die Ökonomie als positivistische Wissenschaft entwickeln konnte. Der Ökonomie gelang es als einziger Sozialwissenschaft, jenen Typ Wissenschaftlichkeit erfolgreich für sich zu reklamieren, der ansonsten nur den Naturwissenschaften zugebilligt wird. Dies war kein Zufall, da sich die Ökonomie seit dem 19. Jahrhundert an den Gleichgewichtsvorstellungen der Newton'schen Physik orientierte (Mirowski 1989). ${ }^{2}$

Der Erfolg der Theorie war nicht nur einer innerhalb des Wissenschaftssystems. Sie war auch politisch ein Erfolg. Die auf der Neoklassik aufbauende Ökonomie ruft den Eindruck der Verfügbarkeit eines Werkzeugkastens zur politischen Steuerung der Wirtschaft hervor. Will man als Politiker Wachstum erhöhen, Inflation bekämpfen oder Arbeitslosigkeit reduzieren, lassen sich aus den Modellen Handlungsempfehlungen ableiten. Dass wir dennoch nicht in der besten aller Welten leben, muss denjenigen zugerechnet werden, die die Objektivität ökonomischer Gesetze nicht einsehen wollen (Böhm-Bawerk 1975) und aus ideologischen Gründen, Unwissenheit oder politischem Opportunismus den Gesetzen der Ökonomie nicht Folge leisten.

Doch ist der Erfolg in Wissenschaftssystem und Politik bereits Beleg für eine zutreffende Konzeptualisierung wirtschaftlicher Prozesse? Ich argumentiere in diesem Artikel, dass auch im kapitalistischen Wirtschaftssystem ein grundlegendes Spannungsverhältnis zwischen wirtschaftlicher und sozialer Rationalität besteht. Dies impliziert, dass sich die Ökonomie nicht „unabhängig von normativen Erwartungen und moralischen Rücksichtnahmen, also einer sittlichen Einbettung“ (Honneth 2011, S. 320 f.) entwickelt. Dieser Einsicht werden jedoch weder Modelle gerecht, die von ausschließlich ökonomischen Handlungsorientierungen in der Wirtschaft ausgehen, noch Modelle, die Wirtschaft und Gesellschaft als zwei getrennte Sphären betrachten.

Ich argumentiere, dass es gerade der normative Charakter der ökonomischen Theorie ist, der verhindert, der Rolle von Normen in der Wirtschaft einen angemessenen Platz einzuräumen. Dafür zeige ich zunächst exemplarisch auf, wie Vorstellungen von Sittlichkeit in das wirtschaftliche Handeln eingehen. Sittliche Einbettung steht dabei beispielhaft für Handlungsorientierungen, die nicht auf ein ökonomisches Kosten-Nutzen-Kalkül reduziert werden können, im wirtschaftlichen Handeln aber dennoch eine Rolle spielen. Neben wertrationalem Handeln gehören zu solchen nicht-ökonomischen Handlungsorientierungen traditionales Handeln, affektuelles Handeln, an Macht orientiertes Handeln

2 Albert O. Hirschman (1986) hat dies unter Rückgriff auf das psychoanalytische Vokabular zum Ausdruck gebracht: Er spricht vom „Physikneid“ der Ökonomen. 
und als selbstverständlich betrachtete kognitive Rahmungen im Sinne der phänomenologischen Tradition. An die Diskussion der sittlichen Einbettung der Wirtschaft schließt sich im zweiten Teil die Frage an, mit welchem konzeptionellen Instrumentarium der Eingang solcher nicht auf Effizienzstreben reduzierbarer Handlungsorientierungen in der Wirtschaft gefasst werden kann. Es bedarf, so der Vorschlag, einer Perspektive auf die Wirtschaft, die diese zugleich in ihrer normativen, sozialen und politischen Konstitution betrachtet.

An dieser Stelle kommt meine Kritik an der ökonomischen Theorie mit einer Kritik an der Soziologie zusammen. Soziologische Theorien funktionaler Differenzierung machen einen konzeptionellen Fehler, der sich kongruent zur ökonomischen Theorie verhält. Sie generalisieren zwar nicht das Modell reiner Effizienzorientierung der Akteure und betrachten somit Gesellschaften nicht als Ökonomien, doch sie betrachten wirtschaftliche Phänomene als getrennt von den normativen Kontexten der Gesellschaft und erklären die Wirtschaft dabei zu einer Sphäre „normfreier Sozialität“, die nach einer Eigenlogik operiert (Habermas 1981). Wirtschaft und Gesellschaft erscheinen so als voneinander getrennte soziale Sphären. Im letzten Teil des Artikels deute ich an, mit welchem Instrumentarium wirtschaftssoziologische Forschung diese Trennung überwinden und damit auch der Sittlichkeit wirtschaftlichen Handelns Raum geben kann. Mein Vorschlag besteht darin, insbesondere mit dem von Pierre Bourdieu entwickelten Konzept des Feldes zu operieren.

\section{Sittliche Handlungsorientierungen in der Wirtschaft}

Wie gehen Vorstellungen von Sittlichkeit in wirtschaftliches Handeln ein? Um diese Frage zu beantworten, ist es sinnvoll, zwischen drei Formen normativer Handlungsorientierungen in wirtschaftlichen Kontexten zu unterscheiden, wobei sich die Unterscheidung an den jeweiligen Konsequenzen für das Marktgeschehen ausrichtet. Ich unterscheide zwischen marktermöglichender Sittlichkeit, marktbegleitender Sittlichkeit und marktbegrenzender Sittlichkeit.

\subsection{Marktermöglichende Sittlichkeit}

Unter marktermöglichende Sittlichkeit lassen sich Phänomene verstehen, die als unverzichtbare Grundlagen der Ausbreitung interessenbasierter Marktbeziehungen zu gelten haben, nicht aber selbst interessenbasiert entstehen können. ${ }^{3}$ Emile Durkheim (1992) verwies mit seinem Konzept der nichtvertraglichen Voraussetzungen des Vertrages auf die normativen Grundlagen von Marktbeziehungen. Verträge, so Durkheim, sind zwingend auf eine sittliche Grundlage angewiesen, denn selbst wenn die Vertragspartner in ihrer Beziehung nur den je eigenen Vorteil suchen, so müssen sie doch darauf vertrauen können, dass sich der Vertragspartner an den geschlossenen Vertrag auch dann halten wird,

3 Amitai Etzioni (1988, S. 199) bringt dies so zum Ausdruck: „,[C]ompetition is not self-sustaining; its very existence, as well as the scope of transactions organized by it, is dependent to a significant extent upon contextual factors [...] within which it takes place“. 
wenn dieser nicht mehr zu seinem Vorteil gereicht. Durkheim sah die Grundlagen hierfür in einer Vertragsmoral, die überhaupt erst die Voraussetzungen für vertragsgesteuerte Marktbeziehungen schaffen würde. Max Weber (1984) beobachtete in seinem Aufsatz über die protestantischen Sekten in Amerika, dass die Mitgliedschaft in bestimmten Sekten Geschäftsbeziehungen mit Fremden erst ermöglichte, weil die Geschäftspartner an einen gemeinsamen ethischen Code gebunden waren. Moral kann also Marktversagen verhindern und dadurch Tauschbeziehungen ermöglichen. ${ }^{4}$

Indirekt ist dieses Problem in der neuen institutionellen Ökonomie (Akerlof 1970; Richter und Furubotn 2003) aufgenommen worden, die zeigt, dass dem arglistigen Verhalten der Akteure durch institutionelle Sicherungen gegengesteuert werden muss. Aus einem strikt interessenbasierten Modell lässt sich die Einrichtung handlungsbindender Institutionen jedoch nicht erklären, da nicht erklärt werden kann, wie die Akteure kollektive Handlungsprobleme überwinden (Knight 1992). Auch wenn der Leviathan als Kollektivgut Vorteile für mich bringen würde, warum sollte ich mich an den Kosten beteiligen? Normative Traditionsbestände, soziale Netzwerke oder institutionelle Strukturen tragen zur Bereitschaft der Marktakteure bei, sich auf riskante Marktbeziehungen einzulassen, indem sie notwendiges Vertrauen stützen. Hier lässt sich von „beneficial constraints“ (Streeck 1997) sprechen. Auch moderne Ökonomien, so die These, sind immer auf ein nicht utilitaristisch zu begründendes Fundament angewiesen. Dabei ist nicht garantiert, dass dieses Fundament auch tatsächlich erhalten bleibt. Unterminiert werden kann es letztlich auch durch Selbstbeschreibungen nach dem Muster der orthodoxen ökonomischen Theorie, die eine Orientierung an individueller Nutzenmaximierung als Erwartung festschreiben und kulturell legitimieren.

Des Weiteren lässt sich aus historischer Perspektive anführen, dass die Ausbreitung von Marktbeziehungen erst dadurch möglich wird, dass der rein interessengeleitete Tausch als eine Form sozialer Beziehung legitimiert ist. Wirtschaftshistorische Befunde zeigen, dass traditionale Gesellschaften Marktbeziehungen strikt reglementierten und begrenzten, weil sie von der gesellschaftszersetzenden Wirkung dieser Form sozialer Beziehungen überzeugt waren (Braudel 1985; Polanyi 1957; Swedberg 2003). So durften Märkte nur außerhalb der Stadtmauern abgehalten werden, waren auf bestimmte Zeiträume begrenzt, und die Beteiligung am Markthandel war auf bestimmte Personengruppen festgelegt, die nicht selten Außenseiter der Gesellschaft blieben. Der Markt ist ,ursprünglich eine Vergesellschaftung mit Ungenossen, also Feinden“ (Weber 1922, S. 385). Erst der Wandel der normativen Bewertung des Markttausches macht die Ausbreitung von Märkten möglich.

Ein drittes, aus der soziologischen Theorietradition gut bekanntes Element marktermöglichender Sittlichkeit lässt sich den Aufsätzen Webers (1984) zur Verbindung von Protestantismus und Kapitalismus entnehmen. Menschen handeln nicht von Natur aus diszipliniert und rational an der Mehrung ihres Wohlstands, sondern wurden erst durch neue religiöse Doktrinen aus ihrem Traditionalismus geradezu herausgedrängt. Die calvinistischen Vorstellungen sittlicher Lebensführung, so Weber, motivierten die Gläubigen

4 Unter den Begriff „marktermöglichende Sittlichkeit“ lässt sich auch das von David Graeber (2011) eingeführte Konzept des „everyday communism“ einordnen, wenngleich dieses weit umfassender ist. 
zu einem Handeln, das zugleich die Herausbildung des modernen westlichen Kapitalismus mit ermöglichte 5 .

Diese integrativen, legitimatorischen und motivationalen sittlichen Grundlagen von Märkten erscheinen heute als so selbstverständliche Teile unserer Lebenswelt, dass ihr moralischer Charakter nicht mehr weiter auffällt. Doch sollte das Bewusstsein historischer Kontingenz davor schützen, Märkte und die darauf zu beobachtenden Handlungsorientierungen als quasi natürliche Phänomene zu deuten. Soweit utilitaristische Handlungsorientierungen bestehen, sind sie vielmehr Teil eines historischen Prozesses moralischer Entwicklung. Die marktermöglichende Sittlichkeit findet keinen systematischen Platz in Theorien rationalen Handelns, sondern erscheint dort entweder als Anomalie (Friedman 1970) oder wird rationalistisch umgedeutet (Ullmann-Margalit 1977).

\subsection{Marktbegleitende Sittlichkeit}

Als marktbegleitende Sittlichkeit lassen sich all die moralischen Vorstellungen bezeichnen, durch die Präferenzen der Marktakteure so beeinflusst werden, dass Nachfrage sich an sittlichen Maßstäben orientiert. Infrage steht hier nicht der Markt als solcher, sondern vielmehr die Konkretisierung der Präferenzen der Akteure. Dies widerspricht zumindest im Bereich von Konsumausgaben in keiner Weise der ökonomischen Theorie, die ja gerade von der Beliebigkeit der Präferenzen der Akteure ausgeht.

$\mathrm{Zu}$ Fragen des moralischen Konsums ist in den letzten Jahren eine umfängliche Forschungsliteratur entstanden (Balsinger 2010; Priddat 1998). Bei Fair-Trade-Produkten etwa - einem kleinen, aber schnell wachsenden Marktsegment - sind Konsumenten bereit, einen höheren Preis für eine Qualität zu bezahlen, die keinerlei funktionalen oder ästhetischen Unterschied im Produkt ausmacht. Bezahlt wird freiwillig für die Besserbezahlung der das Produkt produzierenden Arbeiter oder deren höhere Arbeitssicherheit (Gourevitch 2011; Zick-Varul 2009). Für die Kaufentscheidungen spielen moralische Überzeugungen eine wichtige Rolle (Sunderer und Rössel 2012). Halal-Produkte oder koschere Produkte unterscheiden sich ebenfalls nicht in einer objektivierbaren Produktqualität, sondern in der Berücksichtigung religiöser Vorschriften im Produktionsprozess (van Waarden und van Dalen 2011). Religiöse Doktrinen bestimmen hier Präferenzen für bestimmte symbolische Qualitäten von Gütern und dominieren Kostenerwägungen.

Der Eingang moralischer Prinzipien in Kaufentscheidungen lässt sich weiter verallgemeinern. Bei einer Vielzahl von Produkten erlangen diese ihren Wert aus ihrer symbolischen Verbindung mit moralischen Werten (Beckert 2011). Dass Menschen bereit sind, für einen Ausflug auf das Meer zwecks Beobachtung von Walen Geld zu bezahlen, ist nur denkbar, weil Wale heute kulturell mit Werten wie Freiheit und intakter Natur symbolisch konnotiert sind (Lawrence und Phillips 2004). Antiquitäten werden durch ihre symbolische Verbindung mit Zeiten, Orten oder Personen wertvoll, denen wir eine moralische Bedeutung zuschreiben (Bogdanova 2011). Welche Vorstellungen vom Wohnen wir haben - und also, welchen Wohnraum wir nachfragen -, hängt mit moralischen

5 Allerdings ging Weber davon aus, dass im weiteren Entwicklungsprozess des modernen Kapitalismus solche religiösen Stützen überflüssig würden und stattdessen ein „stahlhartes Gehäuse“ entstünde. (Weber 1984, S. 188). 
Überzeugungen des richtigen Lebens zusammen. ${ }^{6}$ Dass heute in Deutschland über $20 \%$ des Stroms aus erneuerbaren Energien erzeugt werden, ist möglich, weil institutionelle Regulationen politisch durchgesetzt wurden, die diesen Strom subventionieren. Erklären lassen sich die Subventionen nur unter Bezugnahme auf einen über Jahrzehnte andauernden öffentlichen moralischen Diskurs zur Gefährdung von Mensch und Natur durch Atomkraft, zu Nachhaltigkeit, zur Dezentralität der Energieerzeugung usw.

Die Prägung von Präferenzen durch moralische Vorstellungen lässt sich jedoch nicht nur bei Konsumentscheidungen, sondern auch bei Investitionsentscheidungen erkennen. Dies muss stärker überraschen, weil hier der Konkurrenzmechanismus eigentlich die Verfolgung monetärer Maximierungsziele erzwingen müsste. Auf Finanzmärkten spielen in den letzten Jahren ,islamische Investments“ und „soziale Investments“ eine zunehmend größere Rolle. Bei islamischen Investments werden Finanzinvestitionen so strukturiert, dass sie mit dem Zinsverbot des Islams kompatibel sind. Soziale Investments konzentrieren die Finanzanlage auf Firmen, die besondere Anstrengungen bei der Umweltverträglichkeit ihrer Produkte auf sich nehmen, sich entwicklungspolitischen Zielen verschreiben oder aber bestimmte Produkte wie Waffen oder Tabak nicht produzieren. Ein weiteres Beispiel sind Kriegsanleihen (Erster Weltkrieg) und Diaspora-Bonds (Israel und Palästinenser), bei denen Investoren Geld verleihen, nicht weil dies als wirtschaftlich optimale Anlageentscheidung erscheint, sondern weil sie sich als Patrioten moralisch dazu verpflichtet fühlen (Lainer-Vos 2011). Hier werden Gabentausch und Markttransaktion miteinander verbunden. Systematisch betrachtet übersetzen sich religiöse, sozialverantwortliche oder patriotische Werte in spezifische Investitionsentscheidungen, die bei rein materieller Vorteilsorientierung nicht zustande kämen. Mit Robert Frank (1990) können wir hier von „irrational behavior without regret" sprechen. ${ }^{7}$

Der Eingang ethischer Maxime in unternehmerisches Handeln setzt dabei noch nicht einmal zwingend die bewusste Abweichung von Optimierungszielen voraus. Bezieht man die Problematik der Ungewissheit wirtschaftlicher Entscheidungen ein (Beckert 1996), so lassen sich intentional rationale wirtschaftliche Entscheidungen nicht als Optimierungsentscheidungen verstehen, weil genau diese von den Akteuren ex ante gar nicht identifiziert werden könnten. Vielmehr müssen die gewählten Handlungsstrategien als Umsetzung kontingenter Handlungserwartungen gesehen werden (Beckert 2011), in die auch ethische Grundsätze eingehen können.

Ob einzelne Beispiele und Trends für den Eingang moralischer Begründungen in das wirtschaftliche Handeln ausreichen, um von einer „Moralisierung der Märkte“ zu sprechen (Stehr 2007), sei dahingestellt. Wichtig ist hier lediglich, dass ethische Werthaltungen offensichtlich Teil des Markthandelns und von Marktstrukturen sind und somit von

6 So zeigen Hartmut Häußermann und Ingrid Oswald (2001), dass Russen nach 1990 die von ihnen bewohnten Wohnungen noch nicht einmal geschenkt haben wollten. Eigentum an Wohnraum war nach siebzig Jahren Sozialismus schlicht nicht mehr Teil der Moralökonomie der Menschen.

7 In den 70er Jahren wurde von einigen Ökonomen (Sen 1977) das Konzept der Metapräferenzen eingeführt, um Abweichungen von der Verfolgung eigennütziger Interessen zu erklären. Das Konzept ist meines Erachtens wenig erhellend, da unklar bleibt, wie Akteure zwei Präferenzordnungen im Handeln integrieren. 
einer empirischen Theorie wirtschaftlichen Handelns verlangt werden muss, dass sie dem konzeptionell Rechnung tragen kann.

\subsection{Marktbegrenzende Sittlichkeit}

Schließlich kann die sittliche Einbettung wirtschaftlichen Handelns marktbegrenzend sein. Hier lässt sich zwischen der Mikroebene individueller Entscheidungen und der Makroebene institutioneller Strukturen unterscheiden. Märkte können schlichtweg nicht entstehen, wenn es entweder keine Nachfrage oder kein Angebot gibt, weil das Produkt als moralisch verwerflich gilt und die Akteure diese moralische Überzeugung zu ihrer Handlungsmaxime machen. Bekannt ist hierfür das Beispiel von Lebensversicherungen, die im Amerika des 19. Jahrhunderts zunächst keine Nachfrage fanden, weil es als anstößig galt, vom Tod eines nahestehenden Menschen wirtschaftlich zu profitieren (Zelizer 1979). Erfolgreich wurden Lebensversicherungen erst, als sie normativ neu definiert wurden: als verantwortliches Handeln gegenüber Familienangehörigen. Ein anderes Beispiel ist die Verweigerung von Arbeitern im Industrialisierungsprozess, länger als notwendig für die Erwirtschaftung des als ausreichend empfundenen Lebensstandards zu arbeiten (Thompson 1987). Herrscht eine solche traditionalistische Einstellung vor, können keine ökonomisch effizienten Arbeitsmärkte entstehen, da das Arbeitsangebot unelastisch ist. Schließlich kann eine moralisch legitimierte Ablehnung von Profit die Ausbreitung von Märkten begrenzen. Zu denken ist dabei nicht nur an das mittelalterliche Zinsverbot, sondern auch an die Kritik an Spekulationsgewinnen bei Immobilien oder die als ,Heuschrecken“" abgelehnten Aktivitäten von Private Equity Fonds (Münnich 2011).

Die moralisch motivierte Marktbegrenzung findet allerdings hauptsächlich durch Institutionen statt. Bei moralisch anstößigen Märkten, wie denen für Organhandel und Kinderprostitution, ist es ja durchaus nicht so, dass diese nicht entstünden, weil es weder Nachfrage noch Angebot für die Güter und Dienstleistungen gäbe. Viele Dialysepatienten würden eine Niere kaufen, und insbesondere in Ländern der Dritten Welt finden sich Arme, die bereit sind, sich auf dieses Geschäft einzulassen. Dennoch verbieten fast alle Länder den marktförmigen Handel mit Organen (Steiner 2010). Es sind also sanktionsbewehrte Institutionen, durch die marktbegrenzende Sittlichkeit durchgesetzt wird.

Verschiedene politische Philosophen (Anderson 1990; Walzer 1983; Satz 2010) haben sich mit der Frage beschäftigt, weshalb Gesellschaften einige Markttransaktionen verbieten, wenngleich die Präferenzen der Marktteilnehmer diese ermöglichen würden. Die Begründungen verweisen auf die Verletzung moralischer Standards. Debra Satz (2010) schlägt eine Typologie von vier Bedingungen vor, unter denen Markttausch möglicherweise blockiert werden sollte. Die Typologie unterscheidet zwischen individuell oder sozial schädlichen Konsequenzen des Marktes auf der einen Seite und bestimmten Eigenschaften der Marktakteure auf der anderen. Ein Beispiel für individuell schädliche Märkte sind Lebensmittelmärkte, bei denen Preisfluktuationen zu Hunger führen. Sozial schädlich sind Märkte, wenn diese in einer Weise operieren, dass sie „undermine the capacities that a person needs to claim her rights or to participate in society" (ebd., S.95). Märkte sind außerdem moralisch anstößig, wenn die Akteure nur geringe oder hochgradig asymmetrisch verteilte Informationen bzw. Handlungsfähigkeiten haben. Hierunter würden z. B. Organmärkte fallen. Das heißt nicht, dass Märkte mit diesen Eigenschaf- 
ten durchweg unterbunden würden. Doch sie erscheinen als moralisch verwerflich, und Gesellschaften reagieren unter Umständen durch Verbot oder starke Regulierung, um ihren sittlichen Standards im wirtschaftlichen Handeln Geltung zu verschaffen.

Der vermutlich relevanteste Bereich institutioneller Marktbegrenzung in modernen Gesellschaften liegt bei arbeitsrechtlichen Schutzvorschriften und der sozialstaatlichen Sicherung. Es ist vollkommen falsch, die Geschichte des Sozialstaates als funktionalistisches Narrativ zu erzählen, demzufolge der Sozialstaat eingeführt wurde, um die effiziente Funktionsweise des Kapitalismus zu gewährleisten. Eine viel zutreffendere Perspektive auf sozialstaatliche Marktbegrenzung liegt darin, den Sozialstaat als institutionelle Umsetzung normativer Standards von Gerechtigkeit, Freiheit und Gleichheit zu deuten, die durch einen unregulierten Arbeitsmarkt und fehlende soziale Absicherung im Fall der Nichtverwertbarkeit der Arbeitskraft auf dem Markt eklatant verletzt würden. Sozialstaatliche Begrenzungen des Marktes sind das Resultat sozialer Kämpfe, die zu institutionellen Regulierungen führen, durch die Werte der Gesellschaft im Handeln der Wirtschaftsakteure relevant werden können.

Gut sichtbar wird der normative Hintergrund sozialer Rechte in dem epochalen Aufsatz von T. H. Marshall (1950) „Citizenship and Social Class“. Marshall analysiert die Entwicklung des Sozialstaates als Teil einer seit drei Jahrhunderten andauernden Entwicklung von Staatsbürgerrechten. Der Ausbau sozialer Rechte ist nicht bloß eine Reaktion auf funktionale Bedürfnisse des ökonomischen Systems, sondern er vollendet einen politischen Entwicklungsprozess, der die Voraussetzung für die staatsbürgerliche Teilhabe an der Gesellschaft universalisiert, indem die sozialen Grundlagen dieser Teilhabe für alle Staatsbürger gesichert werden.

Gerade anhand sozialstaatlicher Begrenzungen des Marktes kann verdeutlicht werden, dass eine rein effizienzbasierte Erklärung ökonomischer Phänomene unzulässig verkürzend ist. Nicht nur die Präferenzen der Akteure in ihren Marktentscheidungen sind sittlich eingebettet, sondern auch die institutionellen Strukturen der Regulierung der Ökonomie. Weitere Beispiele lassen sich problemlos anführen: Wenn Unternehmen aufgefordert werden, den Frauenanteil in ihren Vorständen zu erhöhen, so werden hier gesellschaftlich verankerte Werte gegen die Präferenzen der Unternehmen durchgesetzt - oder auch nicht. In Konflikten um Nachtflugverbote auf deutschen Flughäfen stoßen wirtschaftliche Interessen auf das beanspruchte Recht der Anwohner, vor Lärmbelästigung geschützt zu werden. Wenn Kapitalerträge mit nur $25 \%$ besteuert werden, Arbeitseinkommen hingegen mit bis zu $45 \%$, dann beruht die Empörung hierüber nicht auf Effizienzargumenten, sondern auf moralischen Überzeugungen der gerechten institutionellen Struktur einer Leistungsgesellschaft.

Gesellschaften, so lassen sich diese Befunde zusammenfassen, verhalten sich widerständig gegenüber ökonomischen Anforderungen, die sich nicht mit den materiellen und ideellen Interessen ihrer organisationsfähigen Akteure vereinbaren lassen. Zwischen der auf Wettbewerb beruhenden Effizienzlogik und den Wertorientierungen der Akteure kommt es zu Friktionen, die sich in sozialen und politischen Konflikten entladen, in denen Akteure Institutionen der Regulation des Wirtschaftssystems so zu gestalten versuchen, dass sie ihren ökonomischen Interessen und moralischen Werten entsprechen (Beckert 2009, S. 188). Der demokratische Kapitalismus lässt sich insofern als eine durch zwei konfligierende Prinzipien charakterisierte Gesellschaftsform beschreiben: einerseits 
orientiert an der Steigerung der Grenzproduktivität, andererseits orientiert an sozialen Bedürfnissen (Streeck 2011). Menschen äußern Widerstand gegen Reformen, die zwar wirtschaftlich effizient sein mögen, die sie aber zugleich als ungerecht empfinden. So konstituiert sich die Ökonomie aus dem Widerstreit von Kriterien der Effizienz und der Gerechtigkeit. „People stubbornly refuse to give up on the idea of a moral economy“ (ebd., S. 6).

Sowohl die Handlungsorientierungen als auch die institutionellen Strukturen der Ökonomie sind geprägt von verschiedenen Werten und politischen und sozialen Auseinandersetzungen, in denen diese Werte institutionell in der Sphäre der Wirtschaft verankert werden. Dabei soll nicht in Abrede gestellt werden, dass die sozialen Belange häufig gegenüber ökonomischen Verwertungsinteressen den Kürzeren ziehen. Die ökonomischen Transformationen der letzten dreißig Jahre lassen sich vornehmlich als Durchsetzung wirtschaftlicher Verwertungsinteressen deuten (Djelic 2006; Streeck 2012). Für die theoretische Konzeptualisierung ist jedoch entscheidend, dass die institutionelle Ordnung der Ökonomie nicht allein effizienztheoretisch erklärt und auch das intentional rationale Handeln der Akteure nicht unabhängig von kulturell verankerten Motiven verstanden werden kann. Die ökonomische Theorie jedoch bleibt den nichtökonomischen Werten der Akteure gegenüber stumm und kann allenfalls - ob zutreffend oder nicht - auf die Wohlfahrtsverluste durch politisch durchgesetzte Marktbegrenzung verweisen.

\section{Die historische Gebundenheit der ökonomischen Theorie}

Wenn dem aber so ist, dann reicht eine sozialwissenschaftliche Perspektive, die sich für die Erklärung ökonomischer Phänomene auf Effizienz als einzigen normativen Maßstab beschränkt, offensichtlich nicht zum Verstehen und Erklären dieser Phänomene aus. Darüber hinaus liefert eine solche Theorie keine Begründung für die Ausrichtung wirtschaftlicher Strukturen am Kriterium der Effizienz. So kommt der ökonomischen Theorie lediglich der Status einer Hilfswissenschaft zu, deren Rat gefragt ist, wenn Entscheidungsalternativen im Hinblick auf die effiziente Verwendung wirtschaftlicher Ressourcen beurteilt werden sollen. ${ }^{8}$

Historisch betrachtet tut man der sich seit dem 18. Jahrhundert entwickelnden modernen ökonomischen Theorie jedoch unrecht, wenn man sie als eine auf ökonomische Effizienz als Ziel gesellschaftlicher Ordnungsprozesse verkürzte Theorie betrachtet. Dies macht der historische Entstehungskontext der Theorie erkennbar. Albert O. Hirschman (1977) hat in seinen brillanten ideengeschichtlichen Untersuchungen gezeigt, dass die Orientierung an Eigeninteresse und ökonomischer Effizienz zeitgenössisch eine moralische Attraktion ausübte, weil Marktfreiheit und Freihandel als Mittel zur Befriedung einer von Bürgerkriegen und Kriegen geprägten Gesellschaft gesehen werden konnten. Noch im 19. Jahrhundert hat John Stuart Mill (1921) die Marktwirtschaft als Befreiung von den Fesseln einer feudalen und aristokratischen Gesellschaft bejubelt. Und Karl Marx (1977) hat die emanzipative Wirkung der entfesselten Märkte geradezu gefeiert,

8 Diese Ratschläge können natürlich am Problem der Ungewissheit von Entscheidungsfolgen scheitern (Beckert 1996). 
weil die kapitalistische Wirtschaft die feudale Ordnung zerstörte und die Rechtsgleichheit als universelles Prinzip etablierte. Selbst in den Theorien der österreichischen Schule und des deutschen Ordoliberalismus aus der Zwischenkriegszeit - also einer Zeit, in der fast ganz Europa von totalitären Regimen beherrscht war - lässt sich das Motiv der Sicherung einer freiheitlichen Ordnung als zentrale Rechtfertigung strikt limitierter staatlicher Intervention in die Wirtschaft erkennen (Röpke 1948).

Hirschman weist in seinen Arbeiten darauf hin, dass die Annahme der Befriedung der Gesellschaft durch den Markt damals umstritten war. Sie wurde aber durchaus auch als emanzipative Gesellschaftstheorie gedeutet. Heute hat sich dieser utopische Gehalt der marktliberalen Theorie, dass die Handlungszwänge des Marktes Wohlstand und Freiheit schaffen, zumindest in den westlichen Industrieländern erschöpft. Dass wirtschaftliches Wachstum das überragende Ziel gesellschaftlichen Handelns sein soll, hat angesichts der sozialen und ökologischen Folgen des Wachstumsimperativs sein utopisches Potenzial eingebüßt. Dass eine Orientierung an wirtschaftlicher Rationalität ein Garant politischer Freiheit ist, wirkt angesichts der durch Märkte erzeugten sozialen Destabilisierung kaum mehr glaubwürdig. Dies sind nicht nur Befunde einer elitistischen Kulturkritik (Horkheimer und Adorno 2003), sondern ist mittlerweile Auffassung eines ernüchterten gesellschaftlichen Mainstreams.

Daraus lässt sich schließen, dass der normative Gehalt der auf (möglichst unbegrenzte) Steigerung der Grenzproduktivität setzenden ökonomischen Theorie selbst einem historischen Wandel in der Gestalt einer Dialektik der Aufklärung unterliegt. Könnte es sein, dass diese Theorie zwar zur Zeit ihrer Entstehung normativ wegweisend war, ihre normative Überzeugungskraft jedoch gerade durch ihren Erfolg bei der Prägung des sozialen Lebens verlor? Folgt man diesem Gedanken, dann hätte die ökonomische Theorie mit ihrem Fokus auf individueller Verfolgung von Eigennutz und dem Markt als zentralem Koordinationsmechanismus einen historischen Index dergestalt, dass diese Theorie im 18. und 19. Jahrhundert einen anderen normativen Rang einnahm als für die heutige Gesellschaft.

Im demokratischen Verfassungsstaat - anders als in feudalen, absolutistischen oder totalitären Gesellschaftsstrukturen - wird die zivilgesellschaftliche Freiheit des Einzelnen gegenüber dem Staat nicht vornehmlich durch die Marktfreiheit, sondern vielmehr durch politische Grundrechte geschützt. Die politischen Grundrechte können nicht nur - wie es den Ordoliberalen vor Augen stand - durch staatliche Willkür, sondern auch durch mangelnden staatlichen Schutz vor den Kräften des Marktes gefährdet werden. Dies gilt zum Beispiel für die soziale Marginalisierung als Konsequenz einer fehlenden marktlichen Verwertbarkeit der eigenen Arbeitskraft, die zu faktischer Ausgrenzung aus dem politischen Gemeinwesen führt. Staatlichen Schutz benötigen die Menschen gerade gegen die aus den Effizienzanforderungen der Märkte entstehenden Risiken, die sie individuell nicht bewältigen können.

Dies deutet auf den Verlust des utopischen Gehalts einer auf die konsequente Detraditionalisierung der Gesellschaft durch Wettbewerb und dadurch erzwungene Rationalisierung von Produktionsprozessen als Mittel zur Steigerung des Wohlstands setzenden Theorie hin. Diskussionen um neue Indikatoren zur Messung von Wohlfahrt, die eine Alternative zum Bruttoinlandsprodukt anbieten (Pennekamp 2011), aber auch das in 
sozialen Protesten von „Wutbürgern“ zum Ausdruck kommende Unbehagen gegenüber einem auf Wachstum verkürzten Fortschrittsbegriff bringen dies zum Ausdruck.

Offensichtlich stehen wir heute vor einer bemerkenswerten intellektuellen Herausforderung. Im historischen Kontext ihrer Entstehung im 18. und 19. Jahrhundert lässt sich die moderne ökonomische Theorie als nicht nur ökonomisch, sondern auch politisch wegweisende Gesellschaftstheorie auffassen. Heute ist sie dies nicht mehr. ${ }^{9}$ Eine Theorie, die sich ausschließlich auf das Kriterium der Effizienzsteigerung kapriziert, wird nicht in der Lage sein, den sich neu stellenden gesellschaftspolitischen Herausforderungen gerecht zu werden. Vielmehr bedarf es einer theoretischen Perspektive auf die Organisation wirtschaftlicher Strukturen, die in komplexerer Weise die sittlichen Ansprüche der Menschen an ein gutes und gerechtes Leben und dessen institutionelle Gewährleistung oder Missachtung in der Organisation der Wirtschaft erklärt.

\section{Wirtschaftliche Ordnung in Theorien funktionaler Differenzierung}

Wie aber sieht eine Theorie aus, die wirtschaftliche Phänomene im Kontext sittlicher Vorstellungen von Gerechtigkeit, Gleichheit, Freiheit und Effizienz beleuchtet? Es wäre völlig falsch zu denken, dass dies einfach ein Umschwenken von der ökonomischen Theorie auf die Soziologie verlangen würde. Denn zum einen gibt es Traditionsbestände im ökonomischen Denken, die wirtschaftliche Phänomene in ihrer normativen und politischen Einbettung untersuchen und an die angeschlossen werden kann. Hierzu zählen in erster Linie die Traditionen der Historischen Schule und der amerikanische Institutionalismus des frühen 20. Jahrhunderts (Reuter 1994) sowie aktuelle heterodoxe Ansätze der Ökonomie (Hodgson und Knudsen 2010).

Zum anderen ist ein Umschwenken auf die Soziologie nicht per se zielführend, weil das soziologische Denken über Wirtschaft im 20. Jahrhundert selbst in weiten Teilen dem Anspruch der Untersuchung von wirtschaftlichen Phänomenen im Kontext normativer Anforderungen nicht gerecht geworden ist. Die einflussreichste Gesellschaftstheorie des 20. Jahrhunderts ist die Theorie funktionaler Differenzierung, die insbesondere die Modernisierungstheorien aus der Mitte des 20. Jahrhunderts informiert hat, dann aber auch die Gesellschaftstheorien etwa von Talcott Parsons, Niklas Luhmann und Jürgen Habermas. Differenzierungstheorien gehen davon aus, dass sich die Ökonomie im Modernisierungsprozess zunehmend zu einer eigenständigen Sphäre ausdifferenziert, die sich von religiösen, politischen, familiären und normativen Kontexten löst. ${ }^{10}$ Die zunehmende Autonomie der modernen Ökonomie wird durch die „Ausbettung“ wirtschaftlichen Handelns aus anderen Handlungszusammenhängen erreicht. Damit werden Wirtschaft und Gesellschaft als voneinander getrennte Sphären betrachtet und der Ökonomie eine Eigen-

9 Die Ironie dabei ist, dass diese normative Ernüchterung auch auf den Erfolg der ökonomischen Theorie in der Gestaltung der sozialen Wirklichkeit zurückzuführen ist.

10 Dies gilt nicht für Parsons, der in dem Begriff der „Interpenetration“ die Verbindung gesellschaftlicher Subsysteme konzeptualisiert. Die Aufteilung der Gesellschaft in Funktionssysteme ist bei Parsons analytisch angelegt, wenngleich man ihm durchaus den Vorwurf einer Essenzialisierung der gesellschaftlichen Subsysteme machen kann (Beckert 1997, S. 230 ff.). 
logik zugesprochen, für deren Beschreibung die neoklassische Wirtschaftstheorie das angemessene Instrumentarium zur Verfügung stellt. Die Soziologie kapriziert sich dann entweder auf Gesellschaftsbereiche „außerhalb“ der Ökonomie oder beschreibt die Austauschprozesse zwischen Wirtschaft und Gesellschaft (Parsons und Smelser 1984).

Dabei ist der Hinweis zwar richtig, dass die Differenzierungstheorie sich insofern von der ökonomischen Theorie unterscheidet, als sie von disparaten Eigengesetzlichkeiten in den verschiedenen sozialen Subsystemen ausgeht, also nicht die Logik der ökonomischen Kalkulation auf alle Gesellschaftsbereiche ausdehnt (Schwinn 2010, S. 206). Doch wird mit der ökonomischen Theorie für die Sphäre der Wirtschaft die Eigenlogik der Ökonomie als allein handlungsprägend angenommen. Bei Luhmann bedeutet Ökonomie „Zahlungen“ und nichts anderes, wobei die Akteure - ganz im Sinn der ökonomischen Theorie - nutzenmaximierend handeln: Sie wollen lieber mehr Geld als weniger Geld. In der Gesellschaftstheorie von Habermas erscheint die Wirtschaft als eine Sphäre „normfreier Sozialität“ und somit als abgetrennt von den in der „Lebenswelt“ verorteten Diskursen gesellschaftlicher Verständigung (Beckert 1991).

Vertreter der Differenzierungstheorie sehen zwar, dass die differenzierten Ordnungen „rein aus ihrer Eigengesetzlichkeit heraus nicht ordnungstauglich sind“ (Schwinn 2010, S.218). Dies gilt, weil Ordnungsbildung auf „sphärenübergreifenden Werten“ (ebd.) beruht, die in jeweils spezifischer Weise in die einzelnen differenzierten Ordnungen eingehen. Hieraus entsteht ein „Zusammenspiel von legitimer Indifferenz und Einbettung“ (ebd., S. 219). ${ }^{11}$ Zugleich jedoch bleibt in der differenzierungstheoretischen Sichtweise „Zielpunkt aller institutionellen Vermittlungen und Innovationen [...] die Garantie der unverwässerten Verfolgung eines Ordnungskriteriums“ (ebd.). Gemeint ist die Eigenlogik des Wirtschaftssystems. Erläutern lässt sich dies am Beispiel der rechtlichen Festlegung, dass Kühlschränke frei von klimaschädlichem FCKW sein müssen. Folge dieser Regulation ist, dass der Unternehmer „nun nicht mehr alle Produktionsfaktoren nach rein ökonomischen Kriterien auswählen (kann), er muss aber innerhalb des nun politisch und rechtlich abgesteckten Rahmens nach rein ökonomischen Maximen handeln“ (ebd., S. 220).

Auffällig an dieser Theoriekonzeption ist der enge Schulterschluss mit der neoklassischen Wirtschaftstheorie. Deren (normative) Konzeptualisierung wirtschaftlichen Handelns und wirtschaftlicher Ordnungsbildung wird anerkannt. Was immer die Differenzierungstheorie der ökonomischen Theorie hinzufügt, bezieht sich auf andere Funktionsbereiche der Gesellschaft oder auf die Bedeutung externer Restriktionen des Wirtschaftssystems. Doch wie im ersten Teil gezeigt, sind externe Restriktionen allenfalls ein Teil der sozialen und politischen Konstitution wirtschaftlichen Handelns. Die von Durkheim und Weber hervorgehobene marktermöglichende Sittlichkeit ist keine sich von außen den Wirtschaftsakteuren aufdrängende Restriktion, sondern inhärenter Bestandteil einer rationalen wirtschaftlichen Ordnung. Ohne ein Minimum an moralischer Zuverlässigkeit der Marktteilnehmer operiert kein Markt. Auch die Präferenzen der Akteure

11 Luhmann (1988) etwa spricht von „Programmen“, durch die das System überhaupt erst entscheidungsfähig wird. Diese werden jedoch von außen an das Wirtschaftssystem herangetragen. Diese Sicht ist daher Ausdruck einer Wirtschaft und Gesellschaft voneinander trennenden Gesellschaftstheorie. 
werden dem Wirtschaftssystem nicht einfach von außen vorgegeben, sondern werden wesentlich im Wirtschaftssystem selbst produziert. Wozu sonst gäbe es Marketing- und Forschungsabteilungen, die Bedürfnisse nach Produkten erzeugen, von denen die Kunden dann meinen, sie immer schon gebraucht zu haben? Schließlich spricht die Problematik der Ungewissheit (Beckert 1996) gegen die Möglichkeit eines ,unverwässerten Ordnungskriteriums“ in der Ökonomie. Gerade die Soziologie der Konventionen (DiazBone 2011) hat gezeigt, dass es, aufgrund von Ungewissheit, wirtschaftliches Handeln ohne dessen Durchdringung durch Konventionen des richtigen und guten Lebens überhaupt nicht geben kann. Wirtschaftliches Handeln beruht auf Rechtfertigungen, die dieses Handeln normativ begründen und sich als Konventionen verfestigen (Thévenot 2007).

Das Markthandeln hat somit einen ganz anderen ontologischen Status als in der ökonomischen Theorie und der Theorie funktionaler Differenzierung: Es ist Teil der sozialen Ordnung und damit auch analytisch nicht sinnvoll als eigene Sphäre beschreibbar. Aufgrund von Ungewissheit und der gleichzeitigen Präsenz verschiedener Konventionen sind verschiedene Formen der Evaluation der Handlungssituation möglich, die im Widerstreit zueinander stehen können und von den Akteuren in Tauschkämpfen und in Auseinandersetzungen um die Gestaltung von Institutionen mobilisiert werden (Diaz-Bone und Thévenot 2010). Die Ökonomie erscheint als Sphäre, in der Kämpfe um die Geltungsmacht verschiedener normativer und kognitiver Prinzipien stattfinden.

In der Differenzierungstheorie wird insinuiert, es gäbe die Ökonomie als eine Handlungssphäre, in der die Akteure allein an Effizienzkriterien orientiert handelten. Genau dies ist aber nicht der Fall. Es gibt kein ökonomisches Handeln unabhängig von normativen und kognitiven Prägungen - und es ist irreführend, von einer „ökonomischen Logik“ zu sprechen, weil diese Prägungen dann nicht mehr als konstitutiv für die Ordnung der Wirtschaft gesehen werden, sondern als ein ihr letztlich äußerlich bleibender Zierrat. Die Differenzierungstheorie übernimmt unkritisch die Prämissen der neoklassischen Ökonomie, in der ebenfalls beliebige Werte nur als externe Restriktionen der nutzenmaximierenden Akteure (lies „Eigenlogik“) in den Modellen erscheinen. ${ }^{12}$

12 Auch Teile der neuen Wirtschaftssoziologie sind nicht besser in der Lage, ökonomische Strukturen als Teil einer sittlichen (und politischen) Ordnung zu beschreiben. Dies mag überraschend erscheinen, verfügt die neue Wirtschaftssoziologie doch mit dem Begriff der Einbettung über ein Konzept, das genau darauf zu zielen scheint. Bei genauerem Hinsehen ist dies jedoch weit weniger eindeutig. Zwar untersucht die Wirtschaftssoziologie wirtschaftliches Handeln als sozial eingebettet. Doch bei der Verwendung des Begriffs der Einbettung etwa bei Mark Granovetter (1985) geht es gerade nicht um die normative Verankerung wirtschaftlichen Handelns, sondern allein um Netzwerkstrukturen und deren Einfluss auf Informationsflüsse, Entscheidungen und daraus resultierende Verteilungen. In seiner Verwendung des Begriffs wendet sich Granovetter, in Absetzung von Parsons, gerade gegen eine Wirtschaftssoziologie, die den soziologischen Beitrag in der Hervorhebung normativer Handlungsorientierungen und entsprechender institutioneller Arrangements erkennt. Damit bleibt der Begriff der Einbettung auf lediglich einen Aspekt der sozialen Verankerung wirtschaftlichen Handelns beschränkt. Etwas anders verhält es sich mit wirtschaftssoziologischen Ansätzen, die den Begriff der Einbettung breiter fassen. Unterschieden wird hier zwischen sozialer, institutioneller, kognitiver und politischer Einbettung wirtschaftlichen Handelns (Zukin und DiMaggio 1990). Wirtschaftliches Handeln erscheint so als kontextabhängig, wobei die Handlungen der Akteure aus den vorherrschenden kognitiven, institutionellen, politischen oder normativen Strukturen erklärt werden. Damit 


\section{Märkte als Felder}

Ein komplexerer Ansatz zur Konzeptualisierung wirtschaftlichen Handelns in seinen Verbindungen zu Werten, aber auch zu kognitiven Rahmungen und politischer Macht ist die Theorie sozialer Felder. Dieser auf die Gestaltpsychologie zurückgehende (Lewin 1997), dann aber insbesondere von Pierre Bourdieu entwickelte Ansatz beschreibt wirtschaftliche Verteilungen als Resultat von Kämpfen in Handlungsfeldern, in denen Akteure aufgrund unterschiedlicher Ressourcenausstattung ungleiche Positionen einnehmen (Bourdieu 1999, 2005). Der Ansatzpunkt ist handlungstheoretisch, indem nicht die „Logiken“ von Systemen beschrieben werden, sondern die Auseinandersetzungen zwischen Akteuren des Wirtschaftssystems in den Vordergrund rücken.

Der Begriff des Feldes bezieht sich auf eine Population von Akteuren, die eine soziale Arena konstituieren, indem sie ihre Handlungen aufeinander beziehen (Fligstein 2001, S. 108). Die Akteure im Feld der Wirtschaft sind Unternehmen und Konsumenten, aber auch der Staat und Intermediäre wie Regulationsbehörden, Gewerkschaften, Lobbygruppen und soziale Bewegungen. Die Handlungen der Akteure sind geprägt durch die Positionen, die sie im Feld einnehmen, und die sozialen Kräfte, die auf sie einwirken und sich in ihrem Habitus manifestieren. Hierzu zählen Netzwerke ebenso wie Institutionen und normative wie kognitive Rahmungen. Durch dieses ,invisible set of forces“ (Fourcade 2007, S. 1022) entsteht eine lokale Ordnung, in der Akteure Handlungsstrategien entwerfen, Erwartungen hinsichtlich des Handelns der anderen Akteure ausbilden und miteinander Positionskämpfe austragen.

Interessant ist der Feldbegriff für die Untersuchung wirtschaftlicher Phänomene, weil er es ermöglicht, ökonomisches Handeln und wirtschaftliche Strukturen aus einer konfliktsoziologisch angelegten handlungstheoretischen Perspektive zu betrachten. Akteure versuchen den Handlungsraum - das Feld - in politischen Kämpfen in ihrem Sinn zu verändern. Dies können Veränderungen der institutionellen Struktur des Feldes sein, Kämpfe um interpretative Vorherrschaft und Veränderungen der Netzwerkstrukturen im Feld (Beckert 2010). Dabei können sie materielle oder ideelle Ziele verfolgen, sie müssen sich mit gegebenen institutionellen Strukturen auseinandersetzen, und normative Standards können selbst als Ressource zur Stabilisierung oder Veränderung bestehender Strukturen eingesetzt werden. Zugleich agieren die Akteure auf der Grundlage von relativ stabilen Dispositionen und Schemata der Wahrnehmung und des Denkens, was im Begriff des Habitus zum Ausdruck gebracht wird. Was als Interesse gilt, konstituiert sich im Feld ausgehend von den Regeln des Feldes, der strukturellen Position der Akteure und ihrem Habitus, beruht jedoch nicht auf einem präsozialen individuellen Nutzen oder einer von den Werten der Akteure unabhängigen „Eigenlogik“ des Systems. Verbindet man das Feldkonzept mit einer pragmatistischen Handlungstheorie, wie dies etwa in der Soziologie der Konventionen vorgeschlagen wird (Diaz-Bone 2011), lässt sich - stärker als in der Feldtheorie bei Bourdieu - die Kreativität und damit die Kontingenz des Handelns der Akteure in den Vordergrund rücken.

werden zwar die Begrenzungen des Netzwerkansatzes überwunden, doch ist mit einer solchen Auflistung noch keinerlei Theorie verbunden. 
Ausgehend von dem Feldkonzept, bleibt das Wirtschaftssystem immer Teil auch der moralischen Ordnung der Gesellschaft. Bourdieu et al. (2002) haben dies etwa in ihrer Studie über den französischen Markt zu Eigenheimen gezeigt, in der sie darlegten, dass die Kaufbereitschaft der Nachfrager von ihrer Gruppenzugehörigkeit und ihren kulturellen Gewohnheiten abhängt und nicht allein vom ökonomischen Kapital und den erwarteten Renditen. Die Verbindung von wirtschaftlichen Strukturen und gesellschaftlichen Wertsphären realisiert sich über soziale und politische Kämpfe, in denen um konkrete Formen der Ausgestaltung des Wirtschaftssystems vor dem Hintergrund von Effizienzanforderungen, politischer Macht und Vorstellungen sozialer Gerechtigkeit, Fairness, Gleichheit, Sicherheit und Freiheit gerungen wird. In diesen Kämpfen wird eine Struktur der Ökonomie institutionalisiert, die sich nicht als Entfaltung einer Effizienzlogik verstehen lässt, sondern als Resultat von Interessenkämpfen, wodurch Märkte sowohl ermöglicht als auch begrenzt werden. Nur so lässt sich die sittliche Einbettung der Wirtschaft, die sich in marktermöglichenden normativen Handlungsorientierungen, den Präferenzen der Akteure und institutionellen Markbegrenzungen zeigt, erklären. Sittliche Einbettung ist Voraussetzung der sozialen Legitimation des Wirtschaftssystems, durch die Systemund Sozialintegration (Lockwood 1970) ermöglicht werden, indem neben wirtschaftlicher Leistungsfähigkeit auch moralische Unterstützung (Offe 1996) generiert wird.

\section{Schluss}

Normative Orientierungen jenseits einer reinen Effizienzorientierung spielen unbestreitbar im wirtschaftlichen Handeln eine Rolle. Doch welche Theorie wird der sittlichen Einbettung wirtschaftlicher Strukturen gerecht? Die ökonomische Theorie unterstellt qua Theorieannahmen eine effizienzorientierte Nutzenmaximierung. Die sittlichen Ansprüche der Akteure gelten als beliebige Geschmäcker, irrationale Traditionsbestände oder politisch zu verantwortende Dysfunktionalitäten. Funktionalistische Differenzierungstheorien in der Soziologie übernehmen in wichtigen Teilen diese Beschreibung des Wirtschaftssystems und werden der Rolle normativer Handlungsorientierungen und konfligierender Kräfte in der Ökonomie damit ebenfalls nicht gerecht.

Dabei wäre es die Aufgabe einer Theorie des wirtschaftlichen Handelns, auch die Bedeutung der sittlichen Einbettung für das wirtschaftliche Handeln und die institutionelle Struktur der Wirtschaft zu beschreiben. Hierfür müsste die Ökonomie als Handlungssphäre sichtbar gemacht werden, in der die Evaluation der Handlungssituation kontingent von Wertkontexten bzw. Rechtfertigungsordnungen der Akteure abhängt. Erkennbar würde so, dass in der Sphäre wirtschaftlichen Handelns verschiedene normative Ansprüche konfliktiv aufeinandertreffen. Wenn dem aber so ist, bleibt eine Theorie, die Handlungsalternativen ausschließlich unter dem Blickwinkel der Effizienz beurteilt, empirisch unzulänglich. Sie kann weder das Bestehen von „beneficial constraints“ (Streeck 1997) noch die Präferenzen der Akteure und ihre Veränderungen zum Gegenstand haben. Sie gibt auch keine Hinweise darauf, wie unter Bedingungen fundamentaler Ungewissheit, also in Situationen, in denen Maximierungsentscheidungen nicht erkennbar sind, dennoch Entscheidungen getroffen werden. Eine solche Theorie bleibt auch normativ unzulänglich, weil sie die Folgen einer rein auf Effizienz ausgerichteten Orga- 
nisation wirtschaftlicher Strukturen selbst wiederum nur unter Effizienzgesichtspunkten zur Kenntnis nehmen kann. Sie verfügt über keinen kritischen Maßstab außerhalb der Effizienzlogik, mit dem wirtschaftliche Phänomene unter Gesichtspunkten der Gerechtigkeit oder des guten Lebens beurteilt werden könnten.

Anders verhält es sich mit der hier vorgeschlagenen konfliktsoziologischen Perspektive. Diese geht von der Gleichzeitigkeit von wirtschaftlichen Interessen an Effizienz und sozialen Interessen an Gerechtigkeit aus und rekonstruiert bestehende wirtschaftliche Konfigurationen und ihre Dynamik aus den Machtpositionen der Akteure im Feld. Soweit sich die Orientierung an der Steigerung von Grenzproduktivität in der Struktur wirtschaftlicher Institutionen vornehmlich durchsetzt - wie dies während der letzten dreißig Jahre der Fall war -, wird diese Entwicklung nicht als ökonomische Eigenlogik reifiziert, sondern als kontingentes Resultat machtdurchsetzter sozialer Auseinandersetzungen verstanden.

Sozial bedeutsam ist dies, weil Theorien selbst eine „materielle Gewalt“ (Marx, vgl. Streeck 2011, S. 3 f.) in sozialen Konflikten sind. Eine Theorie wirtschaftlichen Handelns, die Effizienz als alleinige Evaluationsform wirtschaftlicher Strukturen ausgibt, ist nicht vornehmlich Instrument der Erklärung wirtschaftlicher Sachverhalte, sondern muss ideologiekritisch als Instrument der Verteidigung einer Position im Feld erkannt werden. ${ }^{13}$ Diese performative Rolle von Theorien ist jedoch nicht aus der Binnenperspektive der ökonomischen Theorie zu erkennen, sondern nur aus der Perspektive einer wissenssoziologischen Beobachtung der sozialen Effekte von Theorien. Eine solche Perspektive hält zumindest den Blick offen für die historische Kontingenz der Gestalt der wirtschaftlichen Verhältnisse.

Danksagung: Der Text basiert auf einem Vortrag bei der gemeinsam vom Institut der Deutschen Wirtschaft und dem Max-Planck-Institut für Gesellschaftsforschung veranstalteten Tagung zum Thema „Wie Weltbilder die Wirtschaft prägen“. Für hilfreiche Kommentare bedanke ich mich bei Dominik Enste, Sebastian Kohl und Wolfgang Streeck.

\section{Literatur}

Akerlof, G. A. (1970). The market for „lemons“. Quality uncertainty and the market mechanisms. Quarterly Journal of Economics, 84, 488-500.

Anderson, E. (1990). The ethical limitations of the market. Economics and Philosophy, 6, 179-205.

Balsinger, P. (2010). Making political consumers: The tactical action repertoire of a campaign for clean clothes. Social Movement Studies, 9, 311-329.

Beckert, J. (1991). Die Wirtschaft - ein sittlich neutralisiertes Handlungssystem? Zur Konzeptualisierung des Wirtschaftssystems in der Theorie des kommunikativen Handelns. Berlin: Unveröffentlichtes Manuskript.

Beckert, J. (1996). Was ist soziologisch an der Wirtschaftssoziologie? Ungewißheit und die Einbettung wirtschaftlichen Handelns. Zeitschrift für Soziologie, 25, 125-146.

13 Die Debatte um die Performativität der ökonomischen Theorie (Callon 1998; MacKenzie und Millo 2003) lässt sich genau hier verorten. 
Beckert, J. (1997). Grenzen des Marktes. Die sozialen Grundlagen wirtschaftlicher Effizienz. Frankfurt a. M.: Campus.

Beckert, J. (2009). Wirtschaftssoziologie als Gesellschaftstheorie. Zeitschrift für Soziologie, 38, $182-197$.

Beckert, J. (2010). How do fields change? The interrelations of institutions, networks, and cognition in the dynamics of markets. Organization Studies, 31, 605-627.

Beckert, J. (2011). The transcending power of goods. In J. Beckert \& P. Aspers (Hrsg.), The worth of goods. (S. 106-128). Oxford: Oxford University Press.

Bogdanova, E. (2011). Valuing the past: The constitution of the antiques market in Russia. Dissertation. Köln: Universität Köln.

von Böhm-Bawerk, E. (1975) [1914]. Macht oder ökonomisches Gesetz? Darmstadt: Wissenschaftliche Buchgesellschaft.

Bourdieu, P. (1999). Die Regeln der Kunst. Genese und Struktur des literarischen Feldes. Frankfurt a. M.: Suhrkamp.

Bourdieu, P. (2005). Principles of an economic anthropology. In N. J. Smelser \& R. Swedberg (Hrsg.), The handbook of economic sociology (S.75-89). Princeton: Princeton University Press.

Bourdieu, P., et. al. (2002). Der Einzige und sein Eigenheim. Hamburg: VSA.

Braudel, F. (1985) [1979]. Civilization and capitalism, 15th-18th century. London: Fontana.

Callon, M. (Hrsg.). (1998). The laws of the markets. Oxford: Blackwell Publishers.

Diaz-Bone, R. (2011). Soziologie der Konventionen. Frankfurt a. M.: Campus.

Diaz-Bone, R., \& Thévenot, L. (Hrsg.). (2010). Sociologie des conventions/Soziologie der Konventionen. Themenheft der Online-Zeitschrift Trivium Nr. 5. Paris: Éditions de la maison des sciences de l'homme.

Djelic, M.-L. (2006). Marketization: From intellectual agenda to global policy-making. In M.-L. Djelic \& K. Sahlin-Andersson (Hrsg.), Transnational governance. Institutional dynamics of regulation (S. 53-73). Cambridge: Cambridge University Press.

Durkheim, E. (1992) [1893]. Über soziale Arbeitsteilung. Frankfurt a. M.: Suhrkamp.

Elster, J. (1986). Introduction. In J. Elster (Hrsg.), Rational Choice (S. 1-33). New York: New York University Press.

Etzioni, A. (1988). The moral dimension: Toward a new economics. New York: The Free Press.

Fligstein, N. (2001). Social skill and the theory of fields. Sociological Theory, 19, 105-125.

Fourcade, M. (2007). Theories of markets and theories of society. American Behavioral Scientist, 50, 1015-1034.

Frank, R. H. (1990). Rethinking rational choice. In R. Friedland \& A. F. Robertson (Hrsg.), Beyond the marketplace: Rethinking economy and society (S. 53-87). New York: Aldine de Gruyter.

Friedman, M. (1970). The social responsibility of business is to increase its profits. The New York Times Magazine, September 13, 1.

Gourevitch, P. (2011). The value of ethics: Monitoring normative compliance in ethical consumption markets. In J. Beckert \& P. Aspers (Hrsg.), The worth of goods (S. 86-105). Oxford: Oxford University Press.

Graeber, D. (2011). Debt. The first 5,000 years. New York: Melville House.

Granovetter, M. (1985). Economic action and social structure: The problem of embeddedness. American Journal of Sociology, 91, 481-510.

Habermas, J. (1981). Theorie des kommunikativen Handelns (Bd. 2). Frankfurt a. M.: Suhrkamp.

Häußermann, H., \& Oswald, I. (2001). Wohnungseigentum? Nicht geschenkt! Zur Wohnungsprivatisierung in Russland. Zeitschrift für Soziologie, 30, 65-78.

Hirschman, A. O. (1977). The passions and the interests. Political arguments for capitalism before its triumph. Princeton: Princeton University Press.

Hirschman, A. O. (1986). Rival views of market society. New York: Viking Press. 
Hodgson, G. M., \& Knudsen, T. (2010). Darwin's conjecture. The search for general principles of social and economic evolution. Chicago: University of Chicago Press.

Honneth, A. (2011). Das Recht der Freiheit. Grundriß einer demokratischen Sittlichkeit. Frankfurt a. M.: Suhrkamp.

Horkheimer, M., \& Adorno, T. W. (2003) [1944]. Dialektik der Aufklärung. Philosophische Fragmente. Frankfurt a. M.: Fischer.

Knight, J. (1992). Institutions and social conflict. Cambridge: Cambridge University Press.

Lainer-Vos, D. (2011). Diaspora bonds and the challenge of regulating economic transactions that fuse elements of gift-giving and market exchange. Paper presented at the SASE Conference Madrid 2011.

Lawrence, T. B., \& Phillips, N. (2004). From Moby Dick to Free Willy: Macro-cultural discourse and institutional entrepreneurship in emerging institutional fields. Organization, 11, 689-711.

Lewin, K. (1997) [1951]. Resolving social conflicts \& field theory in the social science. Washington: American Psychological Association.

Lockwood, D. (1970). Soziale Integration und Systemintegration. In W. Zapf (Hrsg.), Theorien des sozialen Wandels (S. 124-137). Köln: Kiepenheuer \& Witsch.

Luhmann, N. (1988). Die Wirtschaft der Gesellschaft. Frankfurt a. M.: Suhrkamp.

MacKenzie, D., \& Millo, Y. (2003). Constructing a market, performing theory: The historical sociology of a financial derivatives exchange. American Journal of Sociology, 109, 107-145.

Marshall, T. H. (1950). Citizenship and social class and other essays. Cambridge: Cambridge University Press.

Marx, K. (1977) [1867]. Das Kapital. Kritik der politischen Ökonomie (Bd. 1). Berlin: Dietz.

Mill, J. S. (1921) [1848]. Grundsätze der politischen Ökonomie. Jena: Fischer.

Mirowski, P. (1989). More heat than light. Economics as social physics. Cambridge: Cambridge University Press.

Münnich, S. (2011). Interest-seeking as sense-making: Ideas and business interests in the new deal. European Journal of Sociology, 52, 277-311.

Offe, C. (1996). Designing institutions in East European transitions. In R. E. Goodin (Hrsg.), The theory of institutional design (S. 199-226). Cambridge: Cambridge University Press.

Parsons, T., \& Smelser, N. (1984) [1956]. Economy and society. A study in the integration of economic and social theory. London: Routledge.

Pennekamp, J. (2011). Wohlstand ohne Wachstum: Ein Literaturüberblick. MPIfG Working Paper, Nr. 11/1. Köln: Max-Planck-Institut für Gesellschaftsforschung.

Polanyi, K. (1957) [1944]. The great transformation. Boston: Beacon Press.

Priddat, B. (1998). Moralischer Konsum. 13 Lektionen über die Käuflichkeit. Leipzig: Hirzel.

Reuter, N. (1994). Der Institutionalismus. Geschichte und Theorie der evolutionären Ökonomie. Marburg: Metropolis-Verlag.

Richter, R., \& Furubotn, E. G. (2003). Neue Institutionenökonomik. Tübingen: Mohr.

Röpke, W. (1948). Die Gesellschaftskrisis der Gegenwart. Erlenbach-Zürich: Rentsch.

Satz, D. (2010). Why some things should not be for sale. Oxford: Oxford University Press.

Schwinn, T. (2010). Wirtschaftssoziologie als Gesellschaftstheorie? Kritische Anfragen aus einer Weber'schen Perspektive. In A. Maurer (Hrsg.), Wirtschaftssoziologie nach Max Weber (S. 199-225). Wiesbaden: VS Verlag für Sozialwissenschaften.

Sen, A. K. (1977). Rational fools: A critique of the behavioral foundations of economic theory. Philosophy and Public Affairs, 6, 317-344.

Smith, A. (1976) [1776]. An inquiry into the nature and causes of the wealth of nations. Chicago: University of Chicago Press.

Stehr, N. (2007). Moral markets: How knowledge and affluence change consumers and products. Boulder: Paradigm Publishers.

Steiner, P. (2010). La transplantation d'organes. Un commerce nouveau entre les êtres humains. Paris: Gallimard. 
Streeck, W. (1997). Beneficial constraints: On the economic limits of rational voluntarism. In R. Hollingsworth \& R. Boyer (Hrsg.), Contemporary capitalism. The embeddedness of institutions (S. 197-219). Cambridge: Cambridge University Press.

Streeck, W. (2011). The crisis in context: Democratic capitalism and its contradictions. MPIfG Discussion Paper, Nr. 11/15. Köln: Max-Planck-Institut für Gesellschaftsforschung.

Streeck, W. (2012). How to study contemporary capitalism? European Journal of Sociology, 53, $1-28$.

Sunderer, G., \& Rössel, J. (2012). Morality or economic interest? The impact of moral motives and economic factors on the purchase of fair trade groceries. International Journal of Consumer Studies, 36, 244-250.

Swedberg, R. (2003). Principles of economic sociology. Princeton: Princeton University Press.

Thévenot, L. (2007). The plurality of cognitive formats and engagements. Moving between the familiar and the public. European Journal of Social Theory, 10, 409-423.

Thompson, E. P. (1987). Die Entstehung der englischen Arbeiterklasse. Frankfurt a. M.: Suhrkamp. Ullmann-Margalit, E. (1977). The emergence of norms. Oxford: Oxford University Press.

Vaihinger, H. (2007) [1911]. Die Philosophie des Als Ob. Saarbrücken: VDM.

van Waarden, F., \& van Dalen, R. (2011). Hallmarking halal. The market for halal certificates: Competitive private regulation. Jerusalem Papers in Regulation \& Governance. Working Paper Nr. 33.

Walzer, M. (1983). Spheres of justice. A defense of pluralism and equality. New York: Basic Books.

Weber, M. (1922). Wirtschaft und Gesellschaft. Tübingen: Mohr.

Weber, M. (1984) [1920]. Die protestantische Ethik I. Eine Aufsatzsammlung. Hamburg: Gütersloher Taschenbücher Siebenstern 53.

Zelizer, V. (1979). Morals and markets: The development of life insurance in the United States. New York: Columbia University Press.

Zick-Varul, M. (2009). Ethical consumption: The case of fair trade. In J. Beckert \& C. Deutschmann (Hrsg.), Wirtschaftssoziologie (S.366-385). Wiesbaden: VS Verlag für Sozialwissenschaften (Sonderheft 49 der Kölner Zeitschrift für Soziologie und Sozialpsychologie).

Zukin, S., \& DiMaggio, P. (1990). Introduction. In S. Zukin \& P. DiMaggio (Hrsg.), Structures of capital. The social organization of the economy (S. 1-36). Cambridge: Cambridge University Press.

Jens Beckert, geb. 1967. Prof. Dr., Direktor am Max-Planck-Institut für Gesellschaftsforschung in Köln. Forschungsschwerpunkte: Wirtschaftssoziologie, soziologische Theorie. Ausgewählte Veröffentlichungen: How do fields change? The interrelations of institutions, networks, and cognition in the dynamics of markets, in: Organization Studies, 2010; Wirtschaftssoziologie als Gesellschaftstheorie, in: Zeitschrift für Soziologie, 2009; (mit P. Aspers, Hrsg.) The worth of goods: Valuation and pricing in the economy, 2011. 